\title{
Dynamics and history of the Milky Way
}

\author{
Amina Helmi \\ Kapteyn Astronomical Institute, University of Groningen, The Netherlands \\ email: ahelmi@astro.rug.nl
}

\begin{abstract}
The structure and dynamics of the Galaxy contain information about both its current workings and its assembly history. I review our understanding of the dynamics of the disk and stellar halo, and sketch how these may be used to unravel how our Galaxy formed.
\end{abstract}

Keywords. Galaxy: dynamics, evolution

\section{Dynamics of the disk(s)}

The structure and kinematics of the Galactic components constrain the mass distribution and history of the Galaxy. For example the vertical dynamics of the thin disk:

- Puts limits on the distribution of mass in the disk, most of which is accounted for by the stars (Kuijken \& Gilmore 1991; Holmberg \& Flynn 2004). Their contribution to the circular velocity (which itself still has large uncertainties, McMillan \& Binney 2009) is roughly half of that required and thus a rounder (dark) component is needed. Also the tilt of the velocity elliposid rules out very flattened oblate halos (Siebert et al. 2008).

- Its coldness has been used to constrain the amount of recent merger events (Toth \& Ostriker 1992). Small dark satellites, which are so abundant in CDM simulations (Springel et al. 2008), do not induce much heating (Font et al. 2001), but mergers of $10-20 \%$ mass ratio can significantly increase the velocity dispersion of the stars.

Stewart et al. (2008) have found that $70 \%$ of dark-matter halos similar to that of the Milky Way $\left(\sim 10^{12} M_{\odot}\right)$ have experienced a merger with an object of $\sim 10^{11} M_{\odot}$ (i.e. mass comparable to the thin disk's) in the last $10 \mathrm{Gyr}$. This would therefore be a plausible origin for the thick disk (Kazanzidis et al. 2008; Villalobos \& Helmi 2009), also if we take into account the age distribution of its stars (Bensby \& Feltzing 2009). However, this merger rate does not account for possible environmental dependences - the Local Group is in a low density region of the Universe, which must imply a smaller chance of encounters. Note as well that this class of mergers are less damaging for gas-rich disks, which is the relevant case for those lookback times (Hopkins et al. 2008).

Other models, besides the minor-merger scenario, have also been proposed for the formation of the thick disk. Abadi et al. (2003) suggest that it may result purely from the accretion of satellites on low inclination orbits, while Brook et al. (2004) find that a thick component might form early on during gas rich mergers (a different but also gaseous formation scenario has been put forward by Bournaud et al., 2008). Recently Schönrich \& Binney (2009) have proposed that the thick disk is composed by stars which have migrated radially via resonant mechanisms from the inner thin disk.

The dynamics of thick-disk stars encode which of these mechanisms has been dominant in the formation of this component. Recently, Sales et al. (2009) have shown that the eccentricity distribution is a particularly powerful discriminant. In all scenarios where the majority of the stars are formed in-situ (minor merger, gas rich mergers or migration), the distribution has a prominent peak at low eccentricity. On the other hand, when the whole disk is built by accretion, the eccentricity distribution is predicted to be flatter, reflecting the range of orbital eccentricities of satellites found in cosmological simulations. 


\section{Dynamics of the stellar halo}

The dynamics of halo stars, and especially of those in streams, can be used to constrain: i) the total mass of the Galaxy and its spatial distribution (e.g. density and shape of the dark matter halo); and ii) the merger history of the Galaxy, as accreted objects will often deposit their debris in this component.

Models of the Sgr streams have yielded conflicting results favouring spherical, oblate or slightly prolate shapes for the Galactic dark halo depending on the set of observations used (Helmi 2004; Johnston et al. 2005; however see Law et al.(2009) who suggest it may be triaxial). Narrow streams are arguably better-suited to derive the gravitational potential in the region probed by their orbits (e.g. Eyre \& Binney 2009). Koposov et al.(2009) have modelled GD-1 and been able to constrain the circular velocity at the Sun to be $\sim 224 \pm 13 \mathrm{kms}^{-1}$, and the shape of the potential (including disk and halo) to have a global flattening $q_{\phi} \sim 0.87$.

Very high-resolution cosmological CDM simulations in combination with semi-analytic models of galaxy formation may now be used to make detailed predictions on the properties of the stellar halo (and in particular the accreted component, De Lucia \& Helmi 2008; Cooper et al., in prep.). The most recent such studies show good agreement with observations, revealing the presence of broad streams such as those from Sgr (typically originated in massive recently objects), and very narrow features, akin the Orphan Stream (Belokurov et al., 2007). Furthermore, in these simulations the very chaotic build up characteristic of the hierarchical structure formation paradigm endows the stellar halo near the Sun with much kinematic substructure (Helmi et al., in prep).

\section{References}

Abadi, M. G., Navarro, J. F., Steinmetz, M., \& Eke, V. R. 2003, ApJ 597, 21

Belokurov, V., et al. 2007, ApJ, 658, 337

Bensby, T., \& Feltzing, S. 2009, arXiv:0908.3807

Bournaud, F., Elmegreen, B. G., \& Elmegreen, D. M. 2007, ApJ 670, 237

Brook, C. B., Kawata, D., Gibson, B. K., \& Freeman, K. C. 2004, ApJ 612, 894

De Lucia, G., \& Helmi, A. 2008, MNRAS, 391, 14

Eyre A. \& Binney, J., 2009, arXiv:0907.0360

Font, A. S., Navarro, J. F., Stadel, J., \& Quinn, T. 2001, ApJ, 563, L1

Helmi, A. 2004, ApJ, 610, L97

Holmberg, J. \& Flynn, C. 2004, MNRAS 352, 440

Hopkins, P. F., Hernquist, L., Cox, T. J., Younger, J. D., \& Besla, G. 2008, ApJ 688, 757

Johnston, K. V., Law, D. R., \& Majewski, S. R. 2005, ApJ, 619, 800

Kazantzidis, S., et al. 2008, ApJ 688, 254

Koposov, S. E., Rix, H.-W., \& Hogg, D. W. 2009, arXiv:090\%.1085

Kuijken, K. \& Gilmore, G. 1991, ApJ 367, L9

Law, D. R., Majewski, S. R., \& Johnston, K. V. 2009, ApJ, 703, L67

McMillan, P. J. \& Binney, J. J. 2009, arXiv:0907.4685

Sales, L. et al. 2009, arXiv:0909.3858 (MNRAS in press)

Schönrich, R. \& Binney, J. 2009, MNRAS 1255

Siebert, A., et al. 2008, MNRAS 391, 793

Springel, V., et al. 2008, MNRAS 391, 1685

Stewart, K. R. et al. 2008, ApJ 683, 597

Toth, G. \& Ostriker, J. P. 1992, ApJ 389, 5

Villalobos, Á. \& Helmi, A. 2009, arXiv:0902.1624 (MNRAS in press) 